Revista del Centro de Investigación de la Universidad La Salle

Vol. 14, No. 54, Julio-Diciembre, 2020: 49-70

DOI: http://doi.org/10.26457/recein.v14i54.2464

\title{
Análisis del gasto social para la prevención y control de la diabetes. Perspectiva futura
}

\section{Analysis of social spending for control and prevention of diabetes. Future perspective}

\author{
María del Rosario Ayala Moreno ${ }^{1}$ \\ Universidad La Salle México (México) \\ María Enriqueta Mancilla Rendón \\ Universidad La Salle México (México) \\ María del Carmen Lozano Arizmendi \\ Universidad La Salle México (México) \\ Arely Vergara Castañeda \\ Universidad La Salle México (México)
}

Recibido: 21 de enero de 2020 Aceptado: 16 de junio de 2020 Publicado: 20 de noviembre de 2020

\section{Resumen}

Este ensayo analítico describe el comportamiento pasado y futuro de los gastos en la prevención y control de la Diabetes Mellitus (DM) en México, y su impacto socio-económico basados en el derecho a la salud. Se analizó la distribución de gastos en salud dirigidos a la atención de la DM (2009-2018) y se desarrolló una proyección de los costos directos e indirectos al 2022, basados en

\footnotetext{
${ }^{1}$ Email: rosario.ayala@ lasalle.mx
}

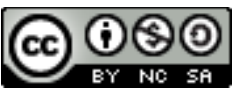

Revista del Centro de Investigación. Universidad La Salle por Dirección de Investigación. Universidad La Salle Ciudad de México se distribuye bajo una Licencia Creative Commons Atribución-NoComercial-Compartirlgual 4.0 
Ayala Moreno, M. R.; Mancilla Rendón, M. E.; Lozano Arizmendi, M. C.; Vergara Castañeda A.

el análisis de series de tiempo de las partidas del Gasto Social de la Administración Pública Federal. El análisis destaca el problema nacional en el abastecimiento de medicamentos, atención oportuna, seguimiento y prevención de los padecimientos crónico-degenerativos, como la DM, observándose gran variabilidad y futuro incierto en la distribución del gasto asignado a prevención y control de la DM. Si bien, la proyección futura de los gastos se realizó a corto plazo, el análisis permite identificar el concepto más desprotegido y urgente en atención, para resolver con mayor eficiencia la atención del paciente con DM. En este sentido resalta la afectación del gasto para tratamiento farmacológico, que tiende a desaparecer en tres años. El análisis sugiere la urgente reorientación en la distribución del gasto que asegure la prevención y sobre todo el tratamiento farmacológico básico para los pacientes.

Palabras clave: Diabetes Mellitus; Prevención de la Diabetes; Control de la Diabetes; Costos de Salud; Gasto social en Salud. 


\section{Abstract}

This analytical essay describes the past and future behavior of expenses in the prevention and control of Diabetes Mellitus (DM) in Mexico, as well as its socio-economic impact based on the right to health. The distribution of health expenditures aimed at DM care (2009-2018) based on the analysis of time series of the Social Expenditure items of the Federal Public Administration was analyzed, and also a projection of direct and indirect costs to 2022 was developed. This analysis highlights the national problem regarding the supplying of medicines, timely care, monitoring and prevention of chronic degenerative diseases, such as DM, suggesting a great variability and an uncertain future in the distribution of the expenditure allocated to prevention and control of DM. Although, the future projection of expenses was carried out in the short term, this analysis allows identifying the most unprotected and urgent concept in care, in order to resolve the care of the patient with DM more efficiently. In this sense, the impact of the expense for pharmacological treatment, which tends to disappear in three years, stands out. Our results suggest the urgent need to redirect the distribution of expenditure that ensures prevention and especially basic pharmacological treatment for patients with DM.

Keywords: Diabetes Mellitus, Prevention of Diabetes, Control of Diabetes, Health costs, Social Expenditure in Health. 


\section{Introducción}

La Diabetes Mellitus (DM) es un problema de salud pública (OMS, 2016), cuyas complicaciones crónicas y agudas aumentan considerablemente los costos de la enfermedad tanto para las instancias de salud como para los pacientes (Cho et. al., 2018). Países como México con gran desigualdad social y sistemas de salud pública precarios, enfrentan una problemática compleja para hacer frente a esta enfermedad. Este ensayo tiene como objetivo analizar la evolución del gasto social en salud durante el periodo 2009-2018, considerando los datos disponibles de la Administración Pública Federal, en particular de aquellos destinados a la atención de la DM en México, y por otra parte desarrollar una proyección de los gastos al 2022, que sugiera los aspectos más urgentes de atender para cubrir con mayor eficiencia la labor de prevención y tratamiento farmacológico de la enfermedad.

\section{Epidemiología de la Diabetes Mellitus}

La DM es considerada una enfermedad de atención prioritaria a nivel mundial, por su alta prevalencia y tasa de mortalidad (OMS, 2016; Cho et. al., 2018). El Global Report on Diabetes 2016 de la Organización Mundial de la Salud (OMS) y el reporte de la red NCD Risk Factor Collaboration, indican que la prevalencia de DM se incrementó de 4.7\% en 1980 a 8.5\% en 2014 (OMS, 2016; Cho et al., 2018), y se estima que para el 2045 existan 693 millones de personas con esta enfermedad (NCD Risk Factor Colaboration, 2016), debido al envejecimiento y al aumento de sobrepeso y obesidad en la población (Cho et. al., 2018; Aguilar-Salinas C, et. al., 2019).

En México, el Instituto Nacional de Salud Pública (INSP), reporta que la prevalencia de DM pasó de $9.2 \%$ en 2012 a $10.3 \%$ en 2018, resaltando que la diabetes y sus principales factores de riesgo son una emergencia de salud pública, que arriesga la viabilidad de los sistemas de salud (ENSANUT, 2016 y 2018). Actualmente, México ocupa el $9^{\circ}$ lugar en DM en el mundo y se estima que para el 2025 ocupe el $7^{\circ}$ lugar (Aguilar-Salinas et. al., 2019). Además, se proyecta un aumento a 15.2\% de la prevalencia ajustada a la población nacional para el año 2035 (Guarugata et. al., 2014). 


\section{El derecho humano a la salud}

El sistema de salud en México se encuentra consagrado en la Constitución Política de los Estados Unidos Mexicanos (CPEUM) y tutela los derechos sociales que brindan bienestar a la sociedad en materia de seguridad social (Constitución Política de los Estados Unidos Mexicanos, 2016). El derecho a la salud es una de las mayores garantías que puede otorgar un gobierno (artículo $4^{\circ}$ ). $\mathrm{Al}$ amparo de la Secretaría de Salud, el gobierno a través de la Administración Pública Federal, en concurrencia con las entidades federativas (Ley General de Salud, artículo 13A y 13B), promueve la salud y ofrece las oportunidades de bienestar físico y mental para todos aquellos que requieran de dicho servicio, cuya visión es procurar que el sistema sea equitativo, integral, sustentable, efectivo y de calidad, dedicando especial cuidado a la prevención de enfermedades (Ley General de Salud, artículo $2^{\circ}$ ) (Ley General de Salud, 2016). Pese a esto, México ocupa en $2^{\circ}$ lugar en obesidad, el $6^{\circ}$ en mayor registro de bajo peso al nacimiento y en el $5^{\circ}$ lugar de mortalidad infantil en el primer año de vida, entre los países de la Organización para la Cooperación y el Desarrollo Económico (OCDE), lo que refleja ciertas deficiencias en sus estrategias de atención a la salud (Carbonel \& Carbonell, 2014).

\section{Impacto socio-económico de la Diabetes Mellitus}

La DM es una enfermedad con serias repercusiones socio-económicas para las familias, que derivan del ausentismo laboral y la pérdida de empleo en la población económicamente activa (Seuring et. al., 2015). Por otra parte, la afección en las instancias de salud, se relacionan con las dificultades para cubrir los gastos de atención ambulatoria e intrahospitalaria para la población. Dicho impacto, puede medirse en términos de costos directos e indirectos (Bommer et. al., 2017). Los costos directos, se relacionan al gasto por atención primaria al paciente (consultas, medicamentos y hospitalización por descompensación) (Aguilar-Salinas et. al., 2015; Bommer et. al., 2017). Estos costos son cuantiosos (Aguilar-Salinas et. al., 2015) y varían desde 242 dólares en México a 11,917 dólares en los Estados Unidos (Ley General de Salud, 2016), no incluyen gastos por complicaciones clínicas (nefropatía diabética, enfermedad cardiovascular, neuropatía, enfermedad vascular periférica), y solo considera la atención en instituciones públicas de salud. 
Ayala Moreno, M. R.; Mancilla Rendón, M. E.; Lozano Arizmendi, M. C.; Vergara Castañeda A.

Por otro lado, los costos indirectos, están relacionados a gastos por mortalidad prematura o discapacidad permanente (Aguilar-Salinas et. al., 2015; Bommer et. al., 2017).

Aunque en México, el $87.7 \%$ de la población con DM se encuentra diagnosticada y con tratamiento (ENSANUT MC, 2016; Centro de Investigación Económica y Presupuestaria (CIEP), 2018), una parte sustancial de los costos de la DM, es cubierta por los pacientes (Aguilar-Salinas et. al., 2015; CIEP, 2018). Es alarmante, que en el mundo casi el 60\% del costo global de la diabetes es sufragado por países de ingresos bajos y medios, con participación sustancial del paciente, afectando la adherencia al tratamiento y dificultando las finanzas de su familia (NCD Risk Factor Colaboration, 2016; Seuring et. al., 2015).

Los costos directos en nuestro país representan el $45 \%$ del monto total destinado a la atención de la DM (3 billones y medio de dólares anuales), donde el 50\% del costo de tratamiento es cubierto por el paciente o su familia, y el resto por las instancias de salud. Por su parte, los costos indirectos, solo han podido ser determinados en el caso de las tres principales instancias de salud pública en México, y representan un total de 4.3 billones de dólares anuales (55\% del monto total de la atención a la DM) (Aguilar-Salinas et. al., 2015).

\section{Composición del gasto social en salud}

El sistema de salud en México está conformado por el sector público (Administración Pública Federal y Estatal) y el sector privado (Ley general de salud, artículo $5^{\circ}$ ) (Ley General de Salud, 2016). Las instituciones públicas como el Instituto Mexicano del Seguro Social (IMSS) y el Instituto de Seguridad Social y Servicios para los trabajadores del Estado (ISSSTE) se financian con las aportaciones del Estado, el patrón y el trabajador, a las cuales se encuentra afiliada la mayor parte de población (46.6\%). En el caso de la atención a los trabajadores de la Secretaría de la Defensa Nacional, la Secretaría de Marina y de Petróleos Mexicanos (0.9\% de los mexicanos), el gobierno federal es quien asume las cuotas de seguridad social (ENSANUT MC, 2016; CIEP, 2018).

Por otra parte, el 13.4\% (IC95\%: 12.0,14.9) de la población en su mayoría de bajos recursos, es beneficiaria de los programas sociales IMSS-Prospera y Seguro Popular (ENSANUT MC, 2016), en el primer caso sin costo del beneficio que obtiene el ciudadano. De esta población poco más de la mitad es atendida por el Seguro Popular (ENSANUT MC, 2016; CIEP, 2018), en donde 
el costo del servicio de salud se determina según la capacidad económica del usuario del servicio (CIEP, 2018).

En México, la inversión del gasto total en salud es del 5.9\% del PIB, del cual el 3\% corresponde al gasto público social y el $2.7 \%$ al sector privado. Datos que están muy alejados del gasto total reportado por países de la OCDE (9.6\% del PIB para el gasto total, con $6.9 \%$ derivado del gasto público) (CIEPa, 2018; Carbonell \& Carbonell, 2014). El gasto social en salud se distribuye anualmente a través del presupuesto federal (CPEUM, artículo 74, fr. IV) (Constitución Política de los Estados Unidos Mexicanos, 2016), a cargo de la Secretaría de Salud para sostener económicamente los programas a nivel nacional (Ley Federal de Presupuesto y Responsabilidad Hacendaria, FPRH, artículo $2^{\circ}$, fr. V) (Diario Oficial de la Federación, 2006). El gasto social se derrama a las entidades públicas y federativas a través del ramo presupuestario 12 (LFPRH, artículo $2^{\circ}$, fr. XXII y XL). Para el presupuesto 2018 se asignó a la Secretaria de Salud una partida de 122,557 millones de pesos (mdp), de los cuales se distribuyó a sector central 10,275 mdp, a los órganos administrativos desconcentrados 85,894 mdp, y a las entidades de tercer nivel 25,764 mdp (Ley Federal de Presupuesto y Responsabilidad Hacendaria, 2006).

El costo de un servicio médico para la diabetes varía según la institución y el lugar de la república en donde se brinda el servicio (CIEPa, 2018). Por ejemplo, el 30\% de los pacientes con diabetes reciben tratamiento en el Seguro Popular, que asciende a 14 mil 991 pesos; mientras que, en el IMSS el tratamiento cuesta 50 mil 776 pesos (CIEPa, 2018). Este costo incluye la cobertura de servicios esenciales como la medición de la glucemia en todos los paciantes con pobre control clínico y se calcula que el sector salud cubrirá también una medición anual de hemoglobina glucosilada (HbA1C) a 205,118 pacientes en tratamiento (enfermedad (CIEPa, CIEPb 2018 con información de la OMS y el Banco de México, 2017).

Estudios anteriores sobre el análisis de proyección para los gastos en diabetes en México muestran una tendencia general a su disminución; sin embargo, no son reportes actuales y aunque estos muestran información relevante, como bien se indica por los autores, es necesario para mayor confiabilidad, validez y pertinencia de las proyecciones actualizar los análisis a los cambios en el ámbito económico y de demanda de los servicios de salud en el país (Arredondo \& De Icaza, 2011; Macías \& Villarreal, 2018). Considerando que la prevalencia de DM en México se incrementará en proporción al crecimiento poblacional, resulta fundamental desarrollar un análisis profundo del 
comportamiento de los gastos en salud asignados en periodos anteriores, y por otra parte generar una proyección de estos para sugerir posibles modificaciones, que puedan ser de utilidad para atender con mayor eficiencia los distintos aspectos: prevención y tratamiento farmacológico para el control de la enfermedad (PEF, 2018-Diario Oficial de la Federación (Gaytán-Hernández et. al., 2017).

\section{Análisis de series de tiempo}

El análisis de series de tiempo es un método cuantitativo que se utiliza para detectar patrones de cambio o permanencia en los datos recolectados a través del tiempo. Posterior al análisis de la serie de tiempo se proyectan los patrones hallados a fin de obtener una estimación para el futuro sin importar la razón por la que va a ocurrir (Pérez-Ramírez, 2007). Los datos considerados para el análisis son observaciones a lo largo del tiempo que, pueden tener una estructura interna (como autocorrelación, tendencia o variación estacional) y son tratados como funciones en una variable de tiempo.

Dentro de los métodos de series de tiempo, existen métodos de suavizamiento simple, los cuales se fundamentan en el supuesto de que existen patrones visibles en la gráfica de la serie de tiempo que pueden ser extrapolados al futuro, esto es, el valor futuro de una variable $\mathrm{Y}$ en el tiempo $t+1$ está en función del valor de la serie de tiempo en el período actual $t$, del período anterior $t-1, \ldots$, en otras palabras:

$$
Y_{t+1}=f\left(Y_{t}, Y_{t-1}, Y_{t-2}, Y_{t-3}, \ldots\right)
$$

Existen diversos métodos utilizados en la literatura que permiten realizar pronósticos para series de tiempo. Entre ellos, el modelo AutoRegresive Integrated MovingAverage (ARIMA) sin estacionalidad (Mauricio, 2007), permite realizar pronósticos a través de datos históricos y errores provocados por el azar. Este método permite analizar datos cuando éstos no muestran un comportamiento estacional a lo largo de cierto periodo de tiempo que pueda considerarse como significativo. 
$\mathrm{Al}$ analizarse el presupuesto en salud, se sabe que históricamente el comportamiento ha sido diferente en cada periodo de tiempo. El método ARIMA suele ser una alternativa sencilla a los modelos econométricos, por ejemplo. En términos simples, un modelo ARIMA puede verse como un filtro que intenta separar la señal del ruido, y la señal se extrapola en el futuro para obtener pronósticos.

La ecuación de pronóstico ARIMA para una serie de tiempo estacionaria es una ecuación lineal en la que los predictores consisten en retrasos de la variable dependiente y/o retrasos de los errores de pronóstico. Un modelo ARIMA no estacional se clasifica como un modelo "ARIMA $(p, d, q)$ ", donde:

$p$ es el número de términos autorregresivos,

$d$ es el número de diferencias no estacionales necesarias para la estacionariedad, y $q$ es el número de errores de pronóstico rezagados en la ecuación de predicción.

La ecuación de pronóstico se construye de la siguiente manera. Primero, y denota la diferencia enésima de $Y$, que significa:

Si $d=0: y_{t}=Y_{t}$

Si $d=1: y_{t}=Y_{t}-Y_{t-1}$

Si $d=2: y_{t}=\left(Y_{t}-Y_{t-1}\right)-\left(Y_{t-1}-Y_{t-2}\right)=Y_{t}-2 Y_{t}-1+Y_{t}-2$

La ecuación general para el pronóstico tiene la siguiente forma:

$$
\hat{y}_{t}=\mu+\phi_{1} y_{t-1}+\cdots+\phi_{p} y_{t-p}-\theta_{1} e_{t-1}-\cdots-\theta_{q} e_{t-q}
$$

El resultado final se presenta considerando intervalos de confianza del $80 \%$ o $95 \%$. 


\section{Materiales y métodos}

\subsection{Análisis de las partidas del Gasto Social en Salud 2009-2018}

Se revisaron datos derivados de las partidas del Gasto Social de la Administración Pública Federal, información disponible para el público general en formato electrónico, del 2009 al 2018. En particular se revisó y analizó el presupuesto federal para el programa social "Prevención y Control de Sobrepeso, Obesidad y Diabetes" (453.58 mdp) (CIEPa, 2018; CIEPb, 2018), para documentar con ello la variación anual de las asignaciones en distintos rubros, entre ellos: gasto social en salud pública, promoción de la salud, prevención y control de enfermedades crónico-degenerativas, prevención y control de sobrepeso, obesidad y DM.

\subsection{Análisis de series de tiempo}

Para analizar la distribución de gastos en salud dirigidos a la atención de la DM, se utilizó el método de series de tiempo, considerando los datos reportados para las partidas anuales del gasto social en salud 2009-2018, en sus diferentes rubros.

En el presente trabajo, se utilizó un modelo ARIMA sin estacionalidad (Mauricio, 2007), para el análisis de las series de tiempo y los pronósticos para el gasto destinado al programa de atención en DM (prevención, control y tratamiento de la enfermedad). Los métodos empleados fueron $\operatorname{ARIMA}(0,0,0)$ y $\operatorname{ARIMA}(0,1,0)$ con niveles de confianza del 80 y $95 \%$, respectivamente.

Para los pronósticos y reportes de resultados se utilizó el software libre R (The R Project forStatistical Computing), el cual es un lenguaje de programación usado frecuentemente para el análisis de series de tiempo (Development Core Team, 2013, pp. 1-3389).

Después de aplicar el análisis de series de tiempo, se proyectan los patrones hallados a cierto periodo de tiempo, en este caso al año 2022.

\section{Resultados}

La Política Pública en Salud ha priorizado el control de la DM, asignando parte del presupuesto federal al programa social "Prevención y Control de Sobrepeso, Obesidad y Diabetes" (453.58 mdp) (CIEPa, 2018; CIEPb, 2018). Este programa de salud es la fusión que se realizó en 2014 con 
el programa enfocado a la prevención de obesidad. La consulta y análisis de las partidas del Gasto Social de la Administración Pública Federal permiten visualizar la evolución que han tenido las asignaciones presupuestales al programa de prevención y control de sobrepeso, obesidad y diabetes en los rubros del gasto social en salud pública, promoción de la salud, prevención y control de enfermedades crónico-degenerativas, prevención y control de sobrepeso, obesidad y DM. Los datos muestran una tendencia a incrementar el gasto social en salud, aunque no es homogénea en todos los años (Tabla 1). 
Ayala Moreno, M. R.; Mancilla Rendón, M. E.; Lozano Arizmendi, M. C.; Vergara Castañeda A.

Tabla 1

Distribución del Gasto Social en México 2009-2018

\begin{tabular}{|c|c|c|c|c|c|c|c|c|c|c|}
\hline Concepto/Año & 2009 & 2010 & 2011 & 2012 & 2013 & 2014 & 2015 & 2016 & 2017 & 2018 \\
\hline $\begin{array}{l}\text { Total Gasto Social } \\
\text { Federal }\end{array}$ & $3,045,478$ & $3,176,332$ & $3,438,896$ & $3,706,922$ & $3,956,361$ & $4,467,225$ & $4,676,237$ & $4,763,874$ & $4,888,892$ & $5,279,667$ \\
\hline $\begin{array}{l}\text { Gasto Social en } \\
\text { Salud Pública }\end{array}$ & 85,036 & 89,893 & 105,314 & 113,479 & 121,856 & 130,264 & 134,928 & 132,216 & 121,817 & 122,557 \\
\hline 1. Sector central & 17,113 & 10,713 & 15,574 & 12,899 & 14,241 & 14,503 & 14,124 & 12,665 & 11,943 & 10,275 \\
\hline $\begin{array}{l}\text { 2.Órganos } \\
\text { administrativos }\end{array}$ & 54,775 & 64,426 & 73,612 & 81,863 & 84,132 & 91,994 & 95,452 & 93,769 & 85,294 & 85,894 \\
\hline 2.1. Comisión & 49,748 & 58,570 & 67,349 & 76,564 & 77,830 & 84,128 & 86,997 & 84,776 & 77,284 & 77,697 \\
\hline $\begin{array}{l}\text { Nacional } \\
\text { de }\end{array}$ & & & & & & & & & & \\
\hline $\begin{array}{l}\text { Protección } \\
\text { Social en } \\
\text { Salud }\end{array}$ & & & & & & & & & & \\
\hline $\begin{array}{l}\text { 3.Entidades de } \\
\text { tercer nivel }\end{array}$ & 13,147 & 14,752 & 16,186 & 18,658 & 23,356 & 23,766 & 25,350 & 25,781 & 24,579 & 25,764 \\
\hline
\end{tabular}


Análisis del gasto social para la prevención y control de la diabetes. Perspectiva futura

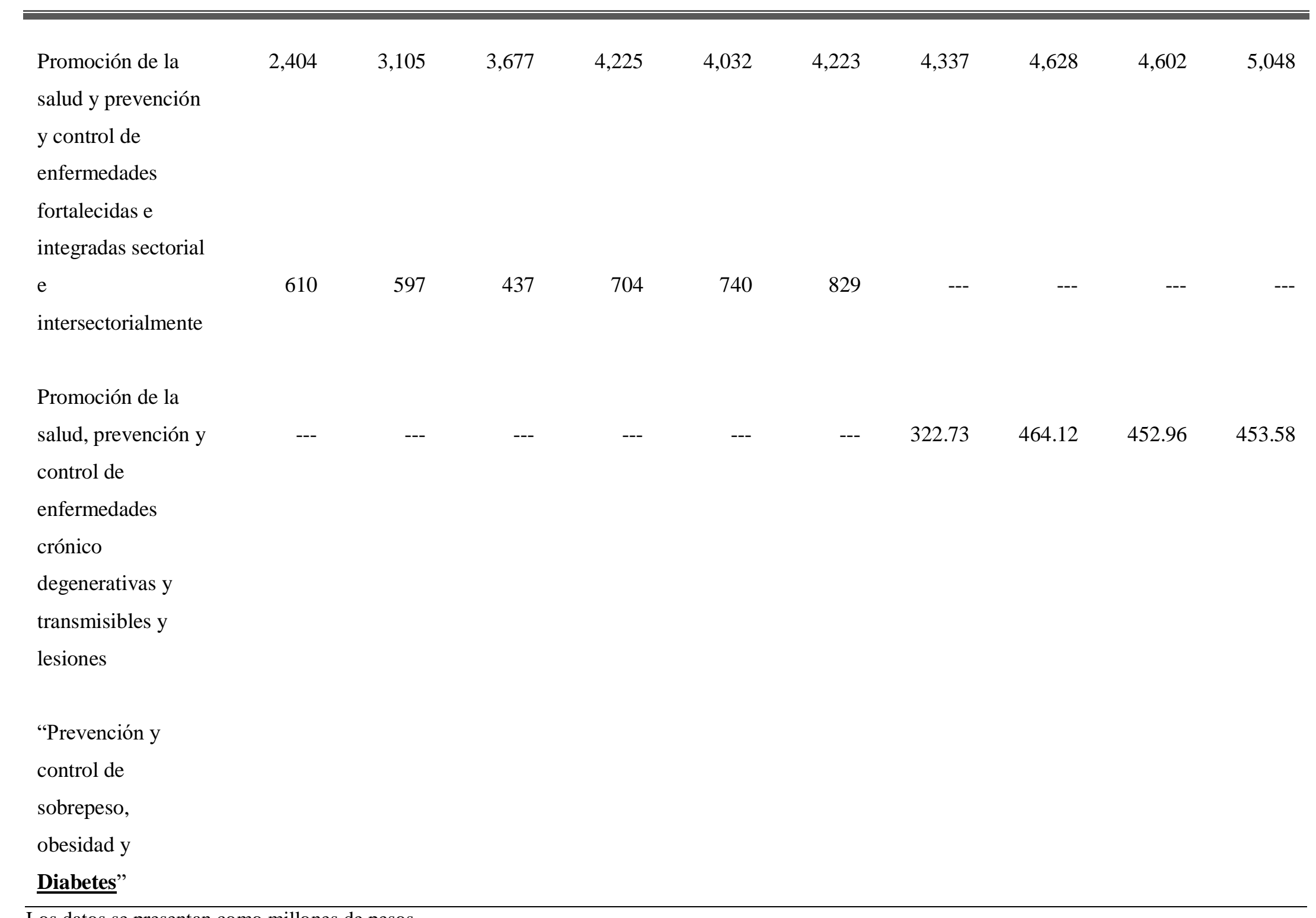

Los datos se presentan como millones de pesos.

Fuente: Elaboración propia con base en el Presupuesto de Egreso de la Federación de distintos años. 
Ayala Moreno, M. R.; Mancilla Rendón, M. E.; Lozano Arizmendi, M. C.; Vergara Castañeda A.

El análisis de la evolución de los datos del Gasto Social Federal muestra una tendencia a incrementarse en el periodo 2009-2018 (Figura 1A). La evolución de los datos del Gasto Social en Salud Pública mostró una tendencia a aumentar considerando el periodo 2009 a 2014, luego del cual se observa un cambio a la baja para el 2016 que se mantiene al 2018 (Figura 1B).
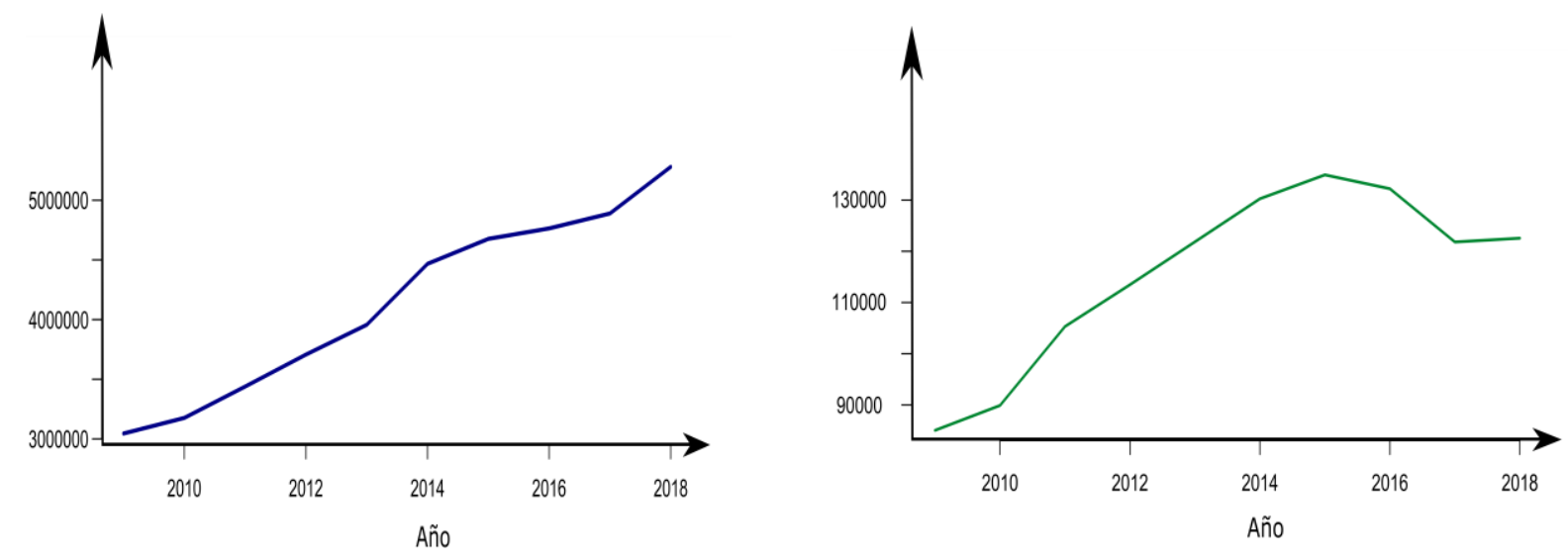

A. Evolución del gasto social federal en México; 2009-

B. Evolución del gasto social en salud de pública en 2018. Elaborada a partir de los datos de la Tabla 1, sobre México; 2009-2018. Elaborada a partir de los datos de el gasto en farmacéuticos como parte del gasto Tabla 1, sobre el gasto social en salud pública, de distribución del gasto social, de acuerdo con el acuerdo con el presupuesto de egreso la federación. presupuesto de egreso la federación.

Figura 1. Evolución del gasto social federal (Figura 1-A) y del gasto social en salud pública (Figura 1-B). Elaborada a partir de los datos de Tabla 1 que muestra la Distribución del Gasto Social en Salud.

En este análisis resalta la tendencia decreciente de la curva para el gasto social federal (Figura 2-A), sugiriendo que la proyección del gasto para tratamiento farmacológico sea prácticamente nula en menos de tres años (Figura 2-B). Por su parte, el análisis de proyección de los costos para promoción de la salud, prevención y control de enfermedades crónicodegenerativas y transmisibles, especificadas en la Tabla 1, tiene un comportamiento aleatorio cuyos grandes aumentos y decrementos, dificulta la proyección de su comportamiento a largo plazo, sugiriendo un panorama incierto en la cobertura de este concepto. Las proyecciones 
para el gasto en promoción y prevención, así como para prevención de otras comorbilidades (sobrepeso y obesidad), se muestran en la Figura 2A y 2 B.

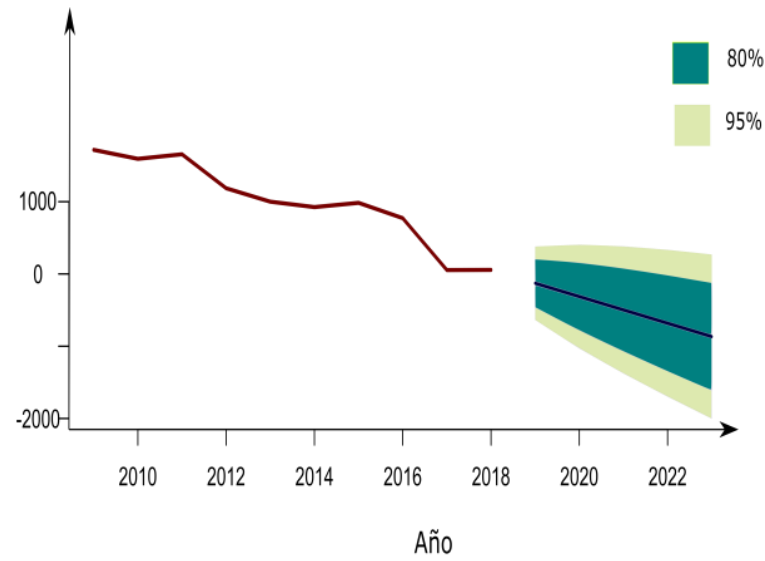

A. Proyección del gasto destinado al tratamiento farmacológico al 2022.

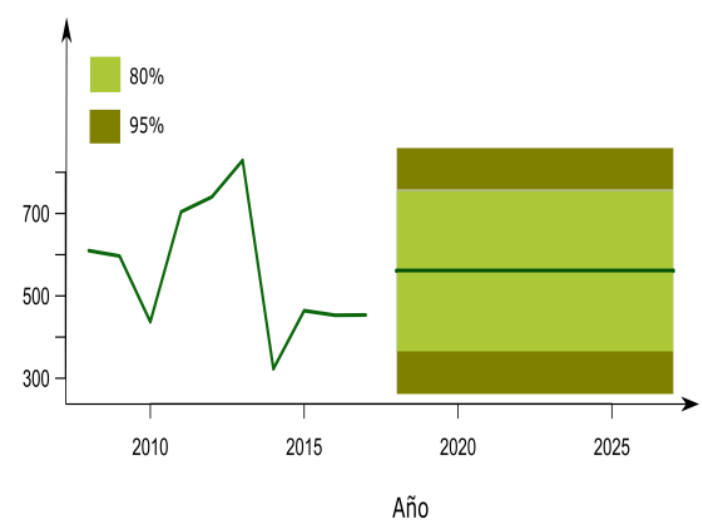

B. Proyección del gasto en promoción y prevención y control de sobrepeso, obesidad y diabetes al 2022.

Figura 2. Proyección al 2022 del gasto destinado al programa de atención en DM en México. Serie de tiempo que muestra la evolución y proyección al 2022 del gasto destinado al programa de atención a diabetes (prevención, control y tratamiento de la enfermedad), basados en la distribución del gasto social en salud, con niveles de confianza del 80 y $95 \%$, respectivamente.

\section{Discusión}

La OMS ha promovido que los países realicen un esfuerzo en atender la salud universal, en el sentido amplio de este concepto y bajo el entendido de que la salud es un derecho humano fundamental, proyecto que plantea alcanzarse para el año 2030. La cobertura universal en salud implica que todas las personas reciban los servicios de salud que necesitan sin tener que pasar penurias financieras para pagarlos. Abarca toda la gama de servicios de salud esenciales de calidad, desde la promoción de la salud hasta la prevención, el tratamiento, la rehabilitación y los cuidados paliativos. Permite además que todos puedan acceder a servicios que atienden las causas más importantes de las enfermedades y la muerte, y asegura que la calidad de esos servicios sea suficientemente buena para mejorar la salud de las personas que 
Ayala Moreno, M. R.; Mancilla Rendón, M. E.; Lozano Arizmendi, M. C.; Vergara Castañeda A.

los reciben. Esta una de las metas que se fijaron los países que adoptaron los Objetivos de Desarrollo Sostenible en 2015, entre ellos México (OMS, 2019). Sin embargo, aunque este compromiso puede ser esperanzador, nuestro país aún está lejos de hacer realidad la cobertura universal en salud. Según el reporte de la OCDE, el gasto sanitario se encuentra entre los más bajos de los países OCDE (5.5\% del PIB) (OCDE, 2019). De acuerdo a los datos recabados de la evolución del gasto social en salud pública, la tendencia en México iba en incremento del 2009 al 2016; sin embargo, de ese año al 2018 el gasto viene a la baja. Lo anterior explica que el país tenga la segunda mayor proporción de gastos de bolsillo de los hogares, que representaron un $41 \%$ adicional del gasto en salud. Los pagos directos excesivos que cubre la población por los servicios médicos generan dificultades financieras, ya que el 5,5\% de los hogares en México experimenta un gasto catastrófico en salud, siendo los hogares pobres los más afectados (OCED, 2019). Todo esto a pesar de que, en México, aproximadamente el 90\% de la población está cubierta por un conjunto básico de servicios de salud, que evidentemente es insuficiente. Si bien, las Instancias de Salud han atendido la implementación de programas que retrasen la aparición de la enfermedad, el impacto de estos no ha generado un efecto positivo suficiente en la prevalencia de DM para verse reflejado en los costos (CIEP, 2018). Es importante considerar que la inversión en gastos en salud debe atender la prevención, promoviendo las conductas saludables en la población, y la detección precoz y el tratamiento en individuos de alto riesgo en un esfuerzo por reducir la carga económica y sanitaria de la DM (Cho et. al., 2018; NCD Risk Factor Colaboration, 2016). Sin embargo, según la información recabada del 2015 al 2018, la inversión del gasto en este rubro ha sido nula.

Por otra parte, las acciones de seguimiento de la enfermedad son deficientes ya que solo contemplan la medición de glucosa en los pacientes con complicación y una sola medición anual de HbA1C como parte del control clínico del paciente, lo cual está muy alejado de la recomendación de la American Diabetes Asociation (ADA, 2019), quien sugiere determinar $\mathrm{HbA} 1 \mathrm{C}$ al menos dos veces al año en pacientes controlados, y cuatro por trimestre cuando existe cambio de terapia o mal control glucémico. Lo anterior sugiere un mayor número de gastos intrahospitalarios por complicación de la enfermedad, tanto para las instancias de salud como para el paciente. 
Según el análisis prospectivo de los datos, la tendencia en los próximos años en la prevención y control de la diabetes no refleja un panorama optimista. Por una parte, las partidas presupuestales son inferiores con relación a las asignadas diez años atrás, y anticipando el aumento de la prevalencia de DM debido al crecimiento poblacional, se anticipa mayor dificultad para la cobertura de los gastos en salud. México gasta poco en salud, en comparación con otros países de la OCDE, además mientras en otros países predomina el gasto público, en México este es minoritario (Constitución Política de los Estados Unidos Mexicanos, 2016). El futuro es más desalentador dados los resultados del ejercicio de proyección del gasto, que resalta la anulación del mismo para tratamiento y la incertidumbre en aquél que es destinado a la prevención y control de la enfermedad.

Parte de la problemática de distribuir adecuadamente el presupuesto a la atención de la DM, radica en que el gasto no considera todos los aspectos que involucran el tratamiento del paciente. En el análisis de la información, según la descripción de objeto del gasto se encuentran conceptos no considerados típicamente en el tratamiento (Rodriguez-Bolaños, 2010), como remuneraciones al personal, prestaciones sociales, pago de estímulos a servidores públicos, gastos de administración, emisión de documentos oficiales, alimentos, utensilios, gastos de construcción y reparación, combustibles, blancos, prendas de protección, herramientas, refacciones, servicios de profesionales, científicos, técnicos, de mantenimiento, de traslado, subsidios, subvenciones, etc., gastos que se erogan en la infraestructura física y mantenimiento, así como en la gestión interna de las Secretarías y para el desarrollo de los programas de salud.

Los gastos vinculados con la capacidad instalada y el recurso humano de la operación implican el uso de infraestructura para atención de otros pacientes y no necesariamente están asociados al costo del tratamiento del paciente diabético, de hecho, es complicado identificar la asignación presupuestal por este concepto. Por ello, se requiere incrementar el monto destinado a la atención de la DM, y analizar los componentes no considerados que suman al tratamiento, además de mejorar el seguimiento de los programas presupuestales destinados control y prevención de la enfermedad, que en algunos casos son deficientes o no existe certeza de su desempeño, en parte debido a la falta de indicadores homogéneos que evalúen su diseño, fiscalización y transparencia (CIEPb, 2018). 
Ayala Moreno, M. R.; Mancilla Rendón, M. E.; Lozano Arizmendi, M. C.; Vergara Castañeda A.

El panorama que se visualiza con la proyección a la baja de los gastos en salud es de considerarse, ya que no es compatible con el aumento esperado en el gasto derivado de los cambios demográficos y epidemiológicos que conllevan aumentos en los costos de atención médica (Macías \& Villareal, 2018) derivados de enfermedades crónico-degenerativas como la DM. De este modo, de no ajustarse un aumento significativo en el gasto en atención en salud, es probable que se sufra de consecuencias financieras derivadas de esta enfermedad con mayor impacto en las instancias de salud de mayor cobertura nacional como el IMSS, SSA e ISSSTE (Arredondo \& Icaza, 2011).

Finalmente, el método de análisis aplicado a la proyección puede tener sus limitaciones, como cualquier otro modelo matemático, pero sobre todo, como mencionan Macías y Villareal (2018), es necesario señalar que para mayor confiabilidad, validez y pertinencia de cualquier análisis de proyección, en primer lugar es necesario que exista disponibilidad de información sobre el diagnóstico y tratamiento de enfermedades crónicas o no transmisibles, así como su costo, derivados de la información pública disponible, bajo un sistema ético de transparencia. Por otra parte, se requiere urgentemente de implementar sistemas de monitoreo eficientes de los costos, que permitan actualizar la medición anual o bianualmente para el manejo de la enfermedad. Esto permitiría ajustar la información de acuerdo a los cambios inflacionarios, costos de insumos y cambios en las tendencias de la demanda de servicios de salud por institución (Arredondo \& Icaza, 2011).

Los análisis de proyección son importantes porque por una parte plantean el comportamiento de la enfermedad como problema de salud pública, especialmente sobre la incidencia y los factores de riesgo para la enfermedad a partir de cambios epidemiológicos esperados (Arredondo \& Icaza, 2011; Gaytán-Hernández et. al., 2017), y por otro lado permiten hacer un análisis de la sostenibilidad de la enfermedad y la adecuación de los gastos en salud destinados a la resolución de un problema de salud pública como la DM.

\section{Conclusiones}

La DM es un problema de salud pública en México que afecta la economía familiar y del Estado. El gasto destinado para su prevención, control y tratamiento ha sido muy variado y 
en el último caso, decreciente. El futuro es incierto en la asignación del gasto para promoción de la salud, prevención y control de la DM, principalmente por la tendencia a desaparecer en corto plazo del gasto para tratamiento farmacológico. Es indispensable analizar y reorientar la distribución del gasto en salud, para generar estrategias eficientes en la atención de los pacientes, centrándose en el derecho humano a la salud.

\section{Agradecimientos}

Agradecemos a la Universidad La Salle el apoyo brindado en la elaboración del presente trabajo.

\section{Referencias}

Aguilar-Salinas, C., Hernández-Jiménez, S., Hernández-Ávila, M., Hernández-Ávila, J.E. (2015) Acciones para enfrentar la diabetes. Colección de Aniversario de la Academia Nacional de Medicina [monografía en internet]. Ciudad de México: Editorial Intersistemas, S.A. de C.V., [Consultado 2019, marzo, 15]:1-25. Recuperado de: https://n9.cl/oGOv

American Diabetes Federation. (2019). Summary of revisions: standards of medical care in diabetes. Diabetes Care, 42(Suppl 1), S4-S6. http://doi.org/10.2337/dc19-Srev01

Arredondo A, De Icaza E. (2011). Costos de la Diabetes en América Latina: Evidencias del Caso Mexicano. Value in Health, (14), S85-S88. https://doi.org/10.1016/j.jval.2011.05.022

Bommer C., Heesemann, E., Sagalova, V., Manne-Goehler, J., Atun, R., Bärnighausen, T., et. al. (2017). The global economic burden of diabetes in adults aged 20-79 years: a cost-of-illness study. Lancet Diabetes Endocrinol, 5(6):423-430.

Carbonell, M. \& Carbonell, J. (2014). La salud en México: perspectiva comparada. En El derecho a la salud: una propuesta para México (31-48). México: Instituto de Investigaciones Jurídicas, Universidad Nacional Autónoma de México

Constitución Política de los Estados Unidos Mexicanos, Diario Oficial de la Federación, publicada el 5 de 1917. Última reforma publicada 27 de enero, 2016. 
Ayala Moreno, M. R.; Mancilla Rendón, M. E.; Lozano Arizmendi, M. C.; Vergara Castañeda A.

Centro de Investigación Económica y Presupuestaria (CIEPa) (2018). Sistema Universal en Salud. Retos de cobertura y financiamiento [monografía en internet]. Ciudad de México: CIEP, A. C. [Consultado 2019, febrero, 22]. Recuperado de: https://n9.cl/WleO

Centro de Investigación Económica y Presupuestaria (CIEPb) (2018). Derecho Universal a la Salud en México: análisis de cobertura y costo. Salud y Finanzas Públicas [monografía en internet]. Ciudad de México: CIEP, A. C. [Consultado 2019, febrero, 22]. Recuperado de: https://ciep.mx/O3vw

Cho NH, Shaw JE, Karuranga S, Huang Y, da Rocha Fernandes JD, Ohlrogge AW, et al. (2018). IDF Diabetes Atlas: Global estimates of diabetes prevalence for 2017 and projections for 2045. Diabetes Res Clin Pract. (138), 271-281. https://doi.org/10.1016/j.diabres.2018.02.023

Development Core Team (2013) R: A language and environment for statistical computing. Vienna, Austria. $R$ Foundation for Statistical Computing, http://www.R-project.org .

Encuesta Nacional de Salud y Nutrición de Medio Camino 2016. (2017) Instituto Nacional de Salud Pública. Secretaría de Salud, México. Recuperado de: https://n9.cl/fb7I

Encuesta Nacional de Salud y Nutrición. Reporte de Resultados 2018. (2020) Instituto Nacional de Salud Pública. Secretaría de Salud, México. Recuperado de: https://bit.ly/2X6E0Hp

Gaytán-Hernández D, Gutiérrez-Enríquez SO, Díaz-Oviedo A, González-Acevedo CE, Miranda-Herrera M, Hernández-Ibarra LE (2017) Escenario futuro de la diabetes mellitus tipo 2 estimado con un modelo de simulación dinámico predictivo. Rev Panam Salud Publica, (41), 1-8.

Guarugata, L, Whiting DR, Hambleton I, Beagley J, Linnenkamp U, Shaw JE. (2014). Global estimates of diabetes prevalence for 2013 and projections for 2035. Diabetes Res Clin Pract, (103), 137-149. https://doi.org/10.1016/j.diabres.2013.11.002

Ley Federal de Presupuesto y Responsabilidad Hacendaria, Diario Oficial de la Federación, publicada el 30 de marzo, 2006.

Ley General de Salud. Diario Oficial de la Federación, México, publicada el 23 de mayo de 1996. Última reforma publicada el 1 de junio, 2016. 
Macías SA, Villarreal PHJ. (2018). Sostenibilidad del gasto público: Cobertura y financiamiento de enfermedades crónicas en México. Ensayos Revista de Economía, 37(1):99-134.

Mauricio, J. A. (2007). Introducción al análisis de series Temporales. Madrid: Universidad Complutense de Madrid. Recuperado de: https://www.ucm.es/data/cont/docs/5182013-11-11-JAM-IAST-Libro.pdf

NCD Risk Factor Collaboration (NCD-RisC). (2016). Worldwide trends in diabetes since 1980: a pooled analysis of 751 population-based studies with 4.4 million participants. Lancet, (387), 1513-1530. https://doi.org/10.1016/S0140-6736(16)00618-8

Pérez-Ramírez, F. (2007). Introducción a las series de tiempo. Métodos paramétricos (1525). Colombia: Universidad De Medellín.

Presupuesto de Egresos de la Federación, Diario Oficial de la Federación, distintos años.

Rodriguez-Bolaños RA, Reynales-Shigematsu LM, Jiménez-Ruiz JA, Juárez-Márquez SA, Hernández-Ávila M. (2010). Costos directos de atención médica en pacientes con diabetes mellitus tipo 2 en México: análisis de microcosteo. Rev Panam Salud Publica, (28), 412-420. http://doi.org/fmbhh3

Seuring T, Archangelidi O, Suhrcke M. (2015). The Economic Costs of Type 2 Diabetes: A Global Systematic. Pharmacoeconomics, (33), 811-831. http://doi.org/10.1007/s40273-015-0268-9

Organización para la Cooperación y el Desarrollo Económicos, OCDE (2019). Health at a Glance 2019, México, ¿Cómo se compara? Recuperado de: https://bit.ly/2ADEQDX

Organización Mundial de la Salud (2016). Global Report on Diabetes 2016, Part 1. Global Burden of Diabetes. WHO, Library Cataloguing-in-Publication Data. Recuperado de: https://n9.cl/rexp

Organización Mundial de la Salud (2019). Cobertura Sanitaria Universal. Recuperado de: https://bit.ly/3fYa7Bw 
Ayala Moreno, M. R.; Mancilla Rendón, M. E.; Lozano Arizmendi, M. C.; Vergara Castañeda A.

A. 